\title{
Optimum Integrated Heterodyne Photoreceiver for Coherent Lidar Applications
}

Farzin Amzajerdian ${ }^{\mathrm{a}}$, Diego Pierrottet ${ }^{\mathrm{b}}$, Upendra Singh $^{\mathrm{a}}$, and Michael Kavaya ${ }^{\mathrm{a}}$

${ }^{a}$ NASA Langley Research Center, Hampton VA 23681

${ }^{\mathrm{b}}$ Coherent Applications, Inc., Hampton VA, 23669

\begin{abstract}
Many coherent lidar applications, particularly airborne and space-based applications, impose stringent power and size constraints while requiring high levels of sensitivity. For this reason, optimization of the lidar heterodyne photoreciever is one of the critical steps in ensuring full utilization of limited resources to achieve the required sensitivity. The analysis of 2-micron heterodyne receivers shows that substantial improvement of the order of $3 \mathrm{~dB}$ can be obtained by proper optimization of the receiver key control parameters and elimination of its parasitic capacitances by integrating the detector, its bias circuit, and the preamplifier on a single substrate. This paper describes analytical steps for defining optimum heterodyne receiver design parameters and development of experimental devices operating at 2-micron wavelength.
\end{abstract}

\section{INTRODUCTION}

Coherent lidar has proven to be a powerful tool for a wide range of remote sensing applications capable of measuring atmospheric wind velocity, turbulence, aerosol concentration, cloud height and velocity, and $\mathrm{CO}_{2}$ concentration. However, most coherent lidar applications continue to demand smaller and more efficient instruments. Therefore, optimization of the lidar heterodyne photoreciever is one of the critical steps in ensuring full utilization of limited resources and achieving the required sensitivity. Analysis of heterodyne photorecievers has projected an improvement of about $3 \mathrm{~dB}$ in lidar sensitivity by reducing the parasitic capacitances associated with the detector and its interfacing pre amplifier and properly adjusting the local oscillator power level [1-2]. This improvement in lidar sensitivity directly translates to a factor of 2 reduction in either the transmitter laser power or the telescope area.

The key areas that must be considered in optimization of a heterodyne photoreceiver are the effects of the detector non-linearity, the parasitic capacitances of the circuit, and the amplifier gain and noise characteristics. This paper describes theses effects, their interactions, and their impact on the photoreceiver performance. Using this analysis, the optimum design parameters for 2-micron heterodyne photoreceivers are defined and the status of the development of a series of experimental devices operating at different bandwidths is reported.

\section{HETERODYNE PHOTORECEIVER MODEL}

The performance of any heterodyne photoreceiver is established by the operating and intrinsic parameters of its detection device and its interfacing preamplifier. Figure 1 shows a typical photoreceiver topology where the detector output current is amplified by a 
transimpedance amplifier. The detector is operated in reverse-biased mode for increased frequency response. The performance of photoreceivers is best described by their Transimpedance Transfer Function (TTF) as opposed to the standard circuit transfer function defined by the circuit response to a rectangular pulse input [2-3]. The TTF simply relates the output voltage of the detector preamplifier to the current generated by the detector upon illumination of an optical radiation for a given circuit topology. The TTF for a transimpedance preamplifier topology, shown in figure 1, can be written in the following general form:

$$
H_{T}(\omega)=\frac{v_{\text {out }}}{i_{\text {in }}}=\frac{-\left(\frac{A_{v}}{1+A_{v}}\right)}{1+j \omega R_{f}\left(C_{f}+\frac{C_{\text {in }}}{1+A_{v}}\right)}
$$

where $A_{v}$ the amplifier open-loop gain, Rf and Cf are the feedback resistance and capacitance, and $\mathrm{Cin}$ is the combined amplifier input capacitance and the detector junction capacitance.

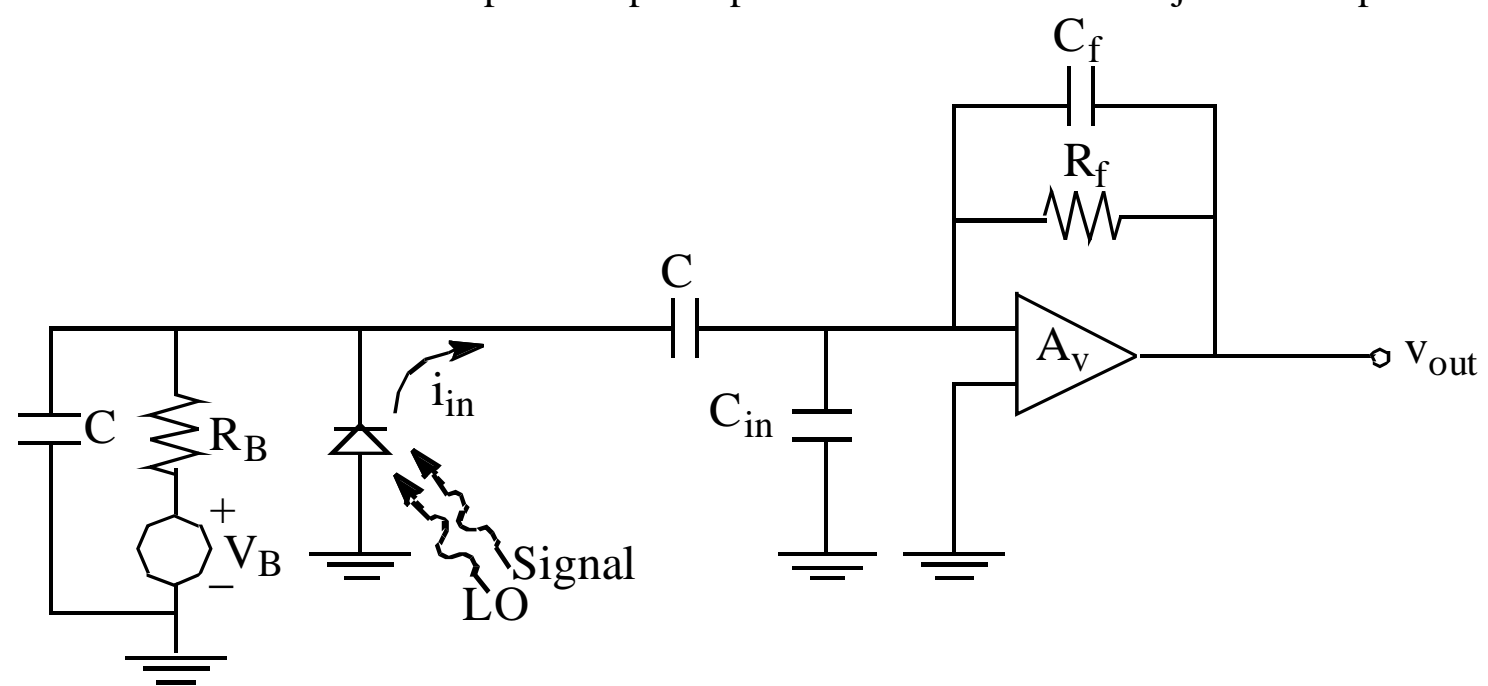

Figure 1. The Optical Heterodyne Receiver Topology.

Using the topology of figure 1, the signal-to-noise ratio (SNR) can be written as

$$
\frac{S}{N}=\frac{2 \rho^{2}\left(1-2 \alpha P_{L O}\right)^{2} P_{L O} P_{s} F_{0}}{\left(2 e \rho\left(1-\alpha P_{L O}\right) P_{L O}+2 e I_{d}+\frac{4 K T_{e}}{R_{f}}+<i_{N_{a m p}}^{2}>B_{e}\right.}
$$

where $\mathrm{P}_{\mathrm{LO}}$ is the applied local oscillator power, $\rho$ and $\alpha$ are the detector responsivity and nonlinearity coefficient, Id is detector bias current, and $\mathrm{i}^{2}{ }_{\text {Namp }}$ is the amplifier total input noise power. The parameter $B_{e}$ is the area under the TTF which is directly related to the receiver bandwidth (BW). The heterodyne signal power reduction due to speckle, turbulence, atmospheric transmission, misalignment and other systematic losses is accounted for by the parameter $\mathrm{F}_{0}[4]$. 


\section{OPTIMIZATION}

The effect of detector non-linearity can be significant and is the limiting factor for the local oscillator power. Ideally, one would apply as much local oscillator power till the detector shotnoise (first term in the denominator) dominates the other noise terms. In this case the coherent detection approaches the shot-noise-limited operation for which the SNR is independent of local oscillator power, given by

$$
\frac{S}{N}=\frac{\rho P_{s} F_{0}}{e B W}
$$

However, in most practical cases, the detector linearity is limited and it starts to saturate before reaching the shot-noise-limited level. In fact, there is an optimum local oscillator power level beyond which any additional increase in local oscillator power results in reduction of the SNR [5]. Figure 2 is a typical repsonsivity curve, showing the detector's non-linear behavior with increasing incident optical power, for an InGaAs detector with an active area diameter of 75 microns operating at 2 microns wavelength [6].

Provided knowledge of the detector responsivity and non-linearity parameters and the preamplifier noise characteristics, the optimum local oscillator power can be obtained from the expression below [7].

$$
\left.P_{\text {LO }}\right|_{\text {opt }}=\frac{1}{2 \alpha}-\frac{1}{2 \alpha}(1+\beta)^{1 / 2}\left[\cos \left(\frac{1}{3} \alpha \tan \sqrt{\beta}\right)-\sqrt{3} \sin \left(\frac{1}{3} \alpha \tan \sqrt{\beta}\right)\right]
$$

where $\beta=\frac{2 \alpha N}{e \rho}$ and $N=2 e I_{d}+\frac{4 K T_{e}}{R_{f}}+\left\langle i_{N_{a m p}}^{2}\right\rangle$

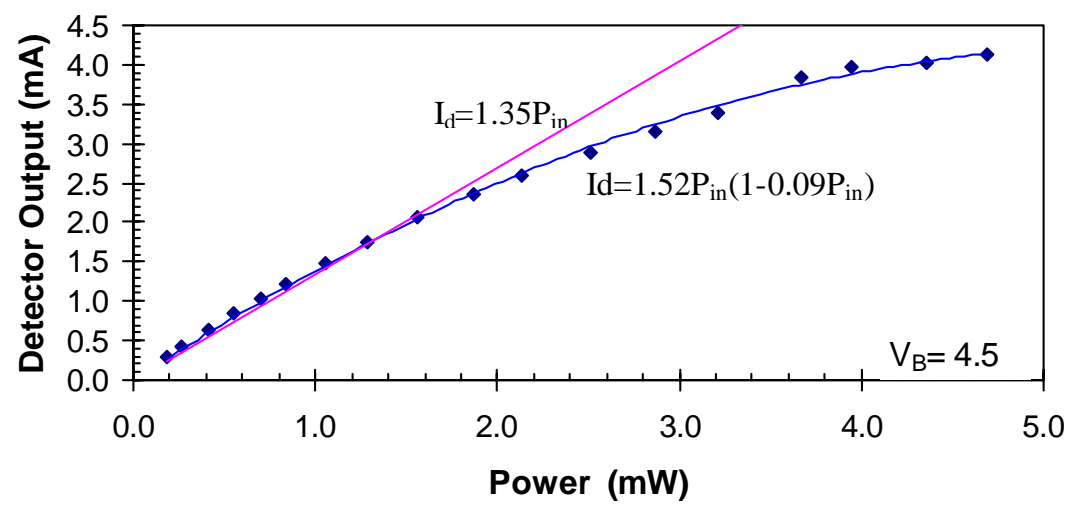

Figure 2. Responsivity of an InGaAs detector at 2 microns wavelength. 
The above expression reveals the significance of both the detector non-linearity and the preamplifier noise power in defining the optimum local oscillator power level. The amplifier noise power in turn is a strong function of the required signal intermediate frequency bandwidth. This is illustrated in Figure 4 where the optimum local oscillator power is plotted as a function of the receiver bandwidth using the parameters of 2-micron InGaAs detector depicted in Figure 2 and typical parameters of commercially available high performance amplifiers. Figure 4 also implies that the common practice of applying as much local oscillator power to achieve shot noise-limited operation can result in unnecessary loss of SNR particularly for narrower bandwidth applications.

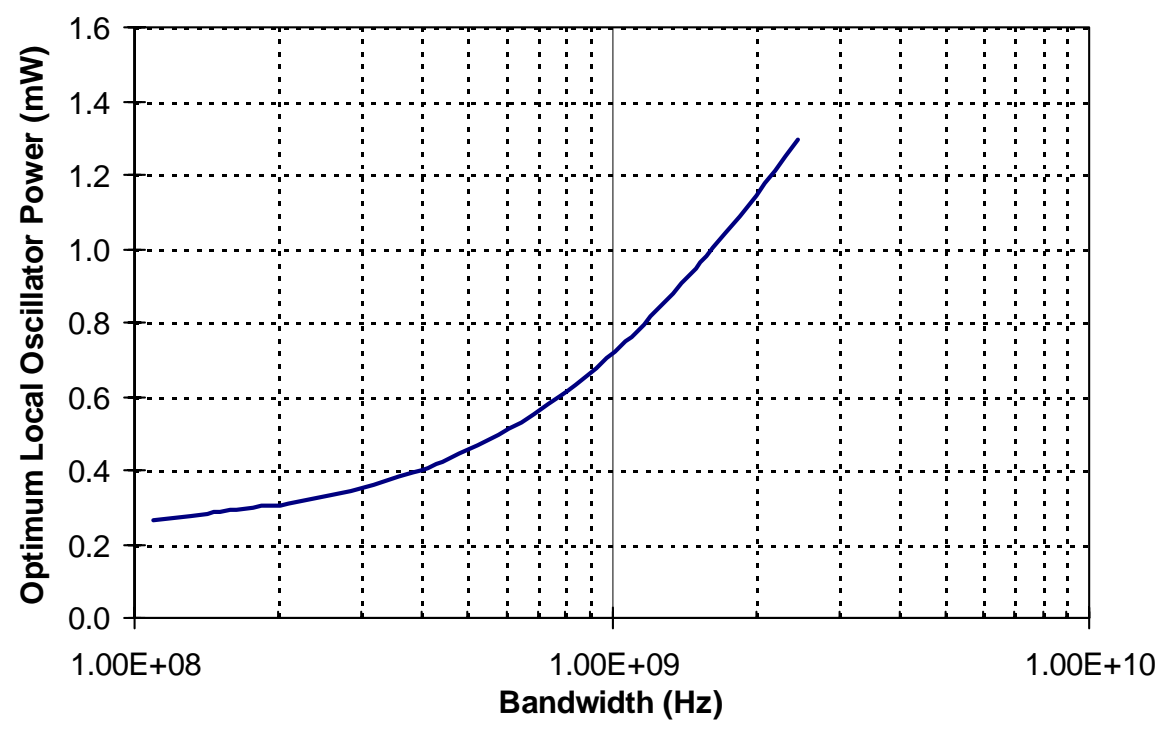

Figure 4. Optimum local oscillator power as a function of operating bandwidth

Another critical step in optimization of a heterodyne receiver is reducing the capacitances associated with the detector and its interfacing pre-amplifier. Any reduction in the receiver circuit capacitance will have a significant impact on the receiver total noise power and operational bandwidth. Since the detector junction capacitance is directly proportional to its active area, it is important to select the smallest detector size possible without introducing excess diffraction and aberration loses due to the focusing elements. The other major limiting factors are the detector linearity and system optical alignment tolerances that will suffer when reducing the detector active area. Once the diameter of the detector active area is specified, the parasitic capacitances associated with the detector and its interface circuits must be minimized. This can be achieved by using die detectors and amplifiers and integrating them on a single substrate thus substantially reducing the parasitic capacitances associated with detector package and amplifier leads. Figure 5 is an example of measured parasitic capacitance for 75-micron diameter InGaAs detector packaged in standard TO can. The shunt capacitance of each detector was measured as a function of applied bias voltage first in its packaged configuration and then the detector elements were removed from their packages and measured gain. As shown in figure 5 , the detector shunt capacitance reduced by about $2.5 \mathrm{X}$. The resultant receiver performance improvement is shown in figure 6 where the normalized SNR for two cases of packaged detector 
with plastic package amplifier and integrated die detector and amplifier. There is more than $2 \mathrm{~dB}$ improvement in SNR for bandwidths greater than $500 \mathrm{MHz}$.
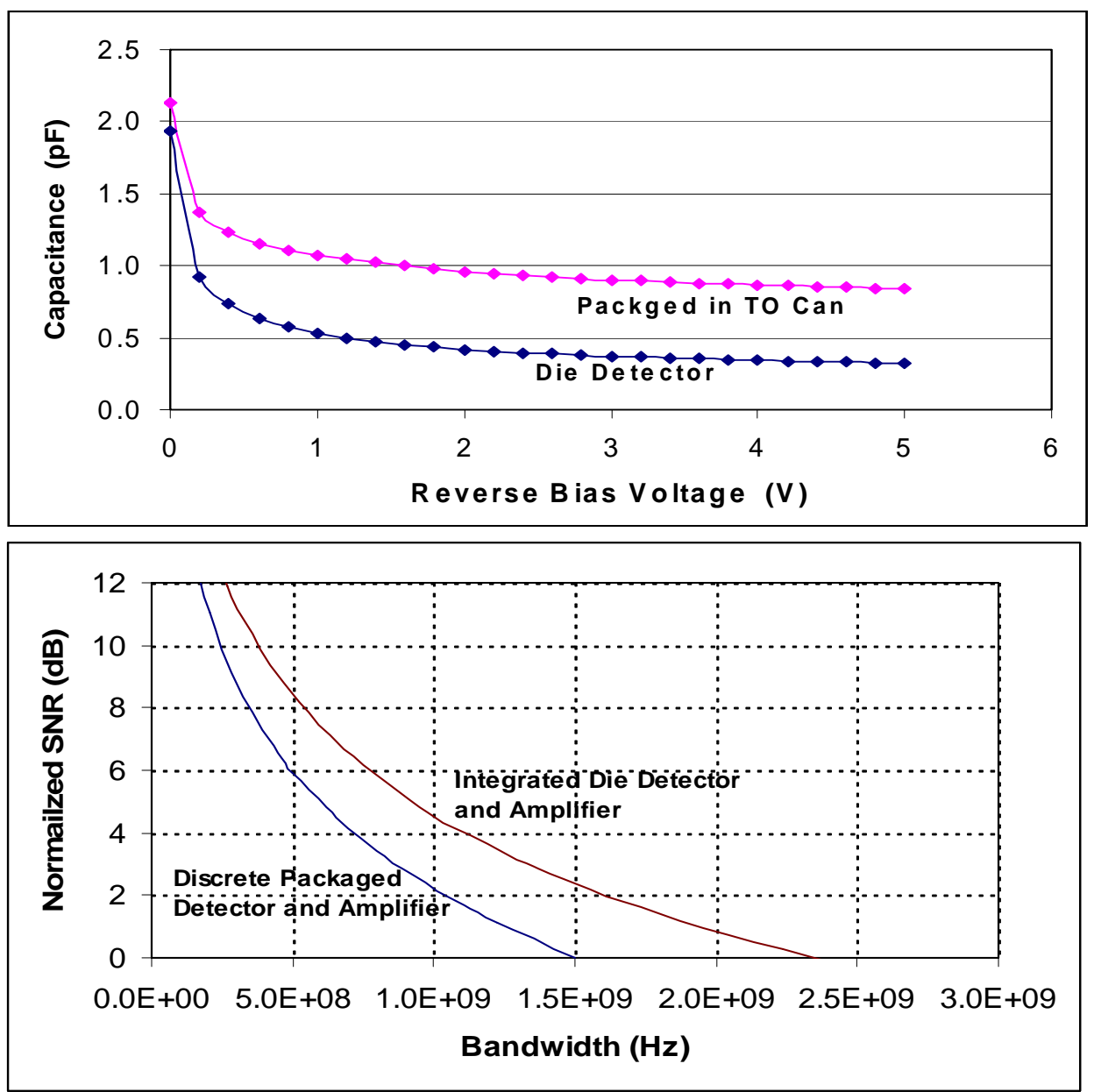

Figure 1

\section{Experimental Photoreceivers}

Several devices with different operational bandwidths are currently being fabricated and tested to demonstrate the improved performance. Figure 7 shows one of these devices using a single amplifier stage. The reviver substrate is about $7 \mathrm{X} 11 \mathrm{~mm}$ and the components including the detector board and amplifier are each about $1 \mathrm{~mm}$ in dimensions. Dual-balanced detectors (save lo power and uses all the signal)

\section{Conclusion}

Many past works have analyzed the effects of the critical photoreceiver parameters and investigated the optimum optical heterodyne detection. It has been shown that there is an optimum local oscillator power level for which the sensitivity of optical heterodyne receiver reaches its maximum value ${ }^{1}$. More recently a closed-form expression defining the optimum local oscillator (LO) power was derived ${ }^{2}$. The object of this paper is to present a full analysis of the combined effects of the detector non-linearity and capacitance, and the amplifier gain and 
noise. This analysis allows for quantifying the impact of various detector and pre-amplifier parameters and their interactions, and provides the mean for true optimization of optical heterodyne receiver. The optimization analysis is then applied to 2-micron heterodyne receivers using InGaAs photodiodes. The analysis of 2-micron heterodyne receivers clearly illustrates the improvements resulting from adjusting the key control parameters of the detector and its interfacing amplifier. The significance of reducing the parasitic capacitances associated with the detector and the pre-amplifier is described, and the analytical approach for optimization of the receiver pre-amplifier is explained. Several experimental receivers with different operational bandwidths have been designed and are being fabricated and tested to show the expected performance improvement of $3 \mathrm{~dB}$ compared with prior art.

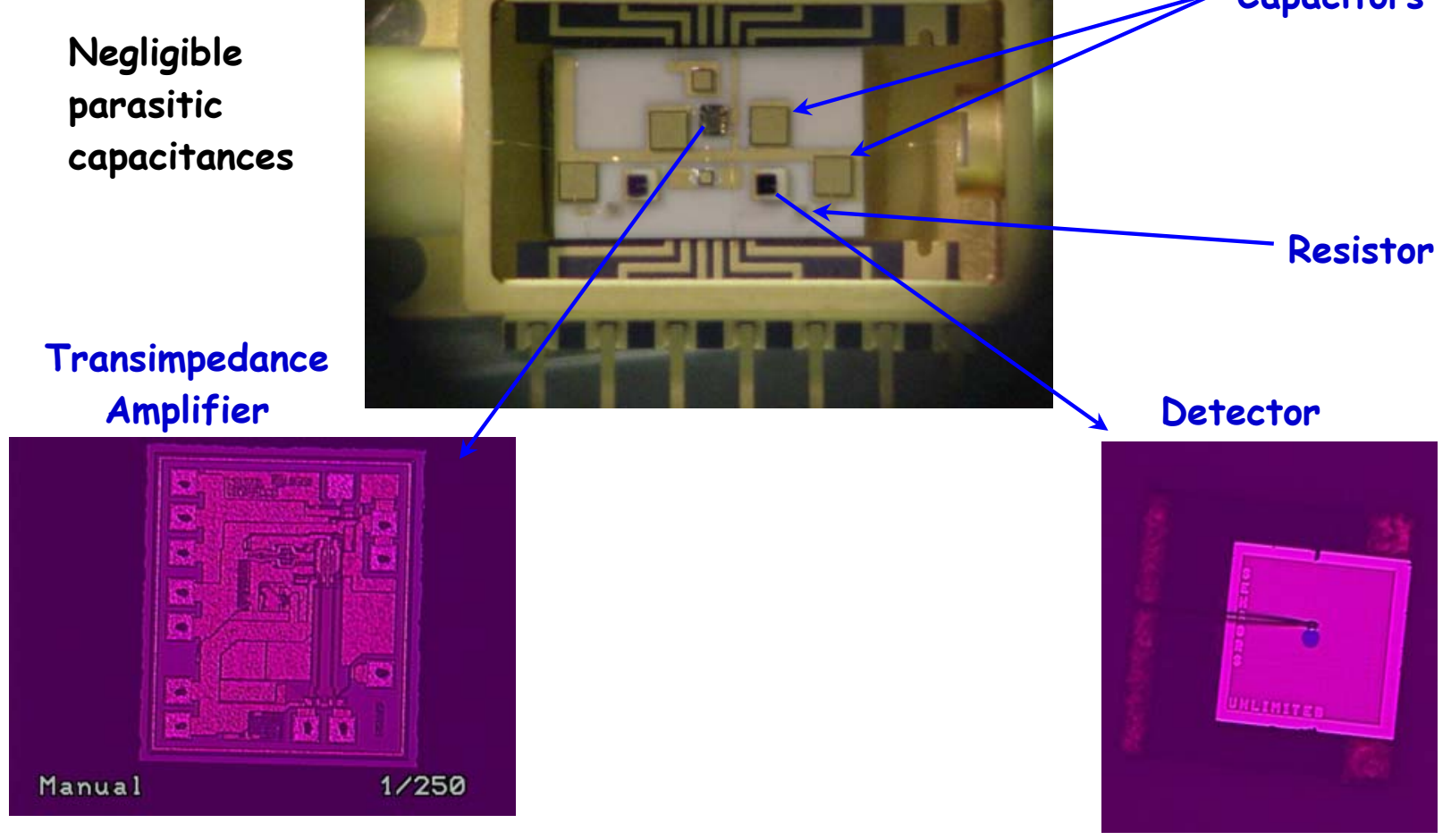

Without fiber pigtails. 


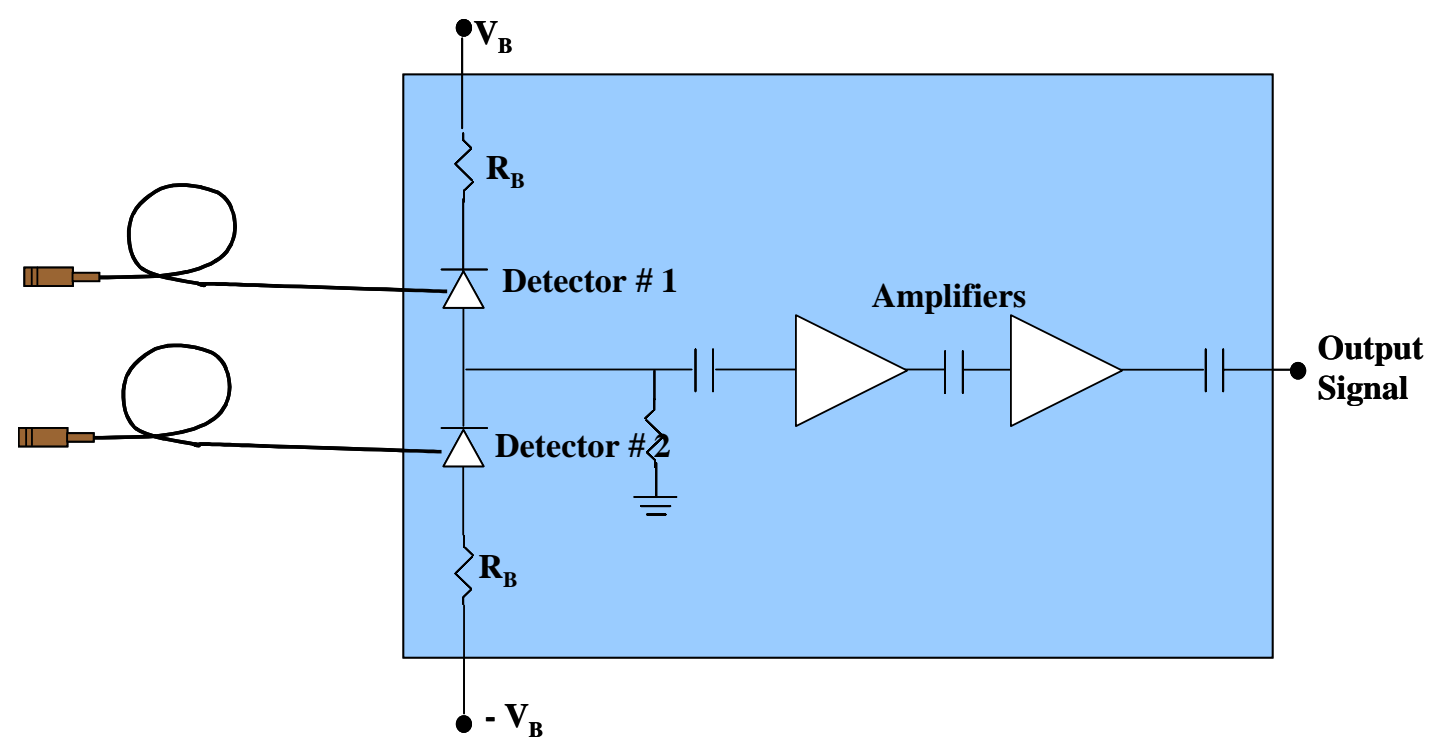

\section{REFERENCES}

1. J. F. Holmes and B. J. Rask, "Optimum optical local-oscillator power levels for coherent detection with photodiodes," Appl. Opt., V. 34, pp 927-933, 1995.

2. F. Amzajerdian, "Improved analytical formulations for optical heterodyne receivers," 11th Coherent Laser Radar Technology and Applications Conference, Great Malvern, UK, July 1-6, 2001.

3. F. Amzajerdian, "Experimental evaluation of InGaAs photodetectors for 2-micron coherent lidars," Conference on Lasers and Electro-Optics, Anaheim, CA, June 2-7, 1996.

4. R. A. Minasian, “Optimum Design of a 4-Gbit/s GaAs MESFET optical preamplifier,” IEEE J. Lightwave Technol., V. 5, pp. 373-379, 1987.

5. H. Statz, P. Newman, I.W. Smith, R. A. Pucel, and H. A. Haus, "GaAs FET device and circuit simulation in SPICE,” IEEE Transactions on Electron Devices, V. ED-34, N. 2, pp. 160-169, 1987. 